\title{
Further evidence of an association between a genetic variant in BMP7 and treatment response to SSRIs in major depressive disorder
}

\author{
Journal of Human Genetics (2013) 58, 568-569; doi:10.1038/jhg.2013.52; published online 23 May 2013
}

\begin{abstract}
Major depressive disorder (MDD) is a common mental disorder with a high lifetime prevalence $(10-20 \%)$, and is a leading cause of disability and mortality. Although antidepressants are often prescribed, the individual response to antidepressants is highly variable, and predictors for treatment efficacy in MDD patients are therefore anticipated to identify the optimal treatment strategy for psychiatrists. Pharmacogenetic and pharmacogenomic (PGx) studies can provide such predictive factors (that is, genetic variants), although none of these have proven useful in the clinical setting for MDD or other psychiatric disorders. One reason for this lack of clinical utility is related to the smaller effect size of each genetic variant on antidepressant (or antipsychotic) efficacy than would be expected in accordance with the pharmacogenetic/pharmacogenomic concept. ${ }^{1}$ Moreover, recent evidence suggests that the genetic variants associated with treatment response are highly polygenic, and heritability is assumed to be $42 \% .^{2}$ These findings indicate that a large number of subjects, as well as independent replication studies with meta-analyses, will be required to derive meaningful results from screening data sets.
\end{abstract}

To address this issue, in the current study, we performed a validation study on genetic associations using the Japanese population and a meta-analysis of single nucleotide polymorphisms (SNPs) detected in the Sequenced Treatment Alternatives to Relieve Depression $\left(\mathrm{STAR}^{\star} \mathrm{D}\right)$ study, ${ }^{3}$ which was performed to determine the treatment efficacy of selective serotonin reuptake inhibitors (SSRIs) in MDD.

The sample comprised 224 MDD subjects treated with SSRIs $($ male $=103$, female $=121$, mean age \pm s.d. $=47.1 \pm 15.0$ years $)$. Detailed information on the clinical phenotypes is described elsewhere. ${ }^{4}$ In the current study, however, we included only patients with a minimum depression severity score of 14 out of 17 items on the Hamilton Depressive Rating scale (HAM-D) and an age range from 18 to 75 years according to the inclusion criteria of $\operatorname{STAR}^{\star} \mathrm{D}^{3}$ Of these patients, 104, 63 and 57 were treated with fluvoxamine, sertraline and paroxetine, respectively, for 8 weeks (monotherapy). The HAM-D scores were assessed in each patient at baseline ( 0 weeks: mean \pm s.d. $=$ $21.0 \pm 4.9)$ and after treatment (8 weeks: mean \pm s.d. $=10.1 \pm 6.1$ ). A responder was defined as a patient who showed a $\geqslant 50 \%$ reduction in HAM-D scores (number of responders $=129$, number of nonresponders $=95$ ), in accordance with previous studies. ${ }^{3}$ SNPs were selected from the $\mathrm{STAR}^{\star} \mathrm{D}$ study, in which the authors examined the association of genome-wide SNPs with citalopram (an SSRI) efficacy. In that study, no SNPs showed evidence at the genome-wide significance level, but the top three SNPs in responders (rs6966038, and rs6127921 and rs809736) ${ }^{3}$ showing a suggestive association with a $P$-value $<1 \times 10^{-5}$ were analyzed in our current study (genotyped by TaqMan SNP genotyping (Life Technologies, Carlsbad, CA, USA) for rs6966038, and PCR-RFLP for rs6127921 and rs809736. Primer pairs are available on request).

Association was assessed using a logistic regression model (dependent variable: responder or non-responder as a categorical variable), with covariates of age, sex, collection site and SNP (additive model: twotailed)..$^{5}$ A meta-analysis was also performed using a fixed-model $\left(I^{2}\right.$ heterogeneity index <50) or random-effect model $\left(I^{2}\right.$ heterogeneity index $\left.\geqslant 50\right)$ on the results

Table1 Association result for SSRI response

Meta-analysis

Frequency of ref allele

$S T A R * D^{a}$

(current study and STAR ${ }^{*} D$ )

\begin{tabular}{|c|c|c|c|c|c|c|c|c|c|c|c|c|c|c|}
\hline \multirow[b]{2}{*}{ CHR } & \multirow[b]{2}{*}{$S N P$} & \multirow[b]{2}{*}{$B P$} & \multirow[b]{2}{*}{$\begin{array}{c}\text { Ref } \\
\text { allele }\end{array}$} & \multicolumn{2}{|c|}{ Frequency of ref allele } & \multirow[b]{2}{*}{$O R$} & \multirow[b]{2}{*}{ s.e. } & \multirow[b]{2}{*}{ P-value } & \multirow[b]{2}{*}{$\begin{array}{c}\text { Closest } \\
\text { gene }\end{array}$} & \multicolumn{2}{|c|}{$S T A R^{*} D^{\mathrm{a}}$} & \multicolumn{3}{|c|}{ (current study and STAR*D) } \\
\hline & & & & Non-responders & Responders & & & & & $\mathrm{P}$ in $\operatorname{STAR}^{*} D$ & OR in $S T A R^{*} D$ & $\mathrm{P}$ (meta) & $O R$ & $1^{2}$ \\
\hline 7 & rs6966038 & 156880398 & $\mathrm{G}$ & 0.076 & 0.071 & 0.876 & 0.377 & 0.72 & UBE3C & $4.65 E-07$ & 1.64 & 0.332 & 1.33 & 65.5 \\
\hline 20 & rs6127921 & 55638750 & C & 0.60 & 0.48 & 0.586 & 0.207 & 0.0098 & $B M P 7$ & $3.45 E-06$ & 0.61 & $1.11 \mathrm{E}-07$ & 0.60 & 0 \\
\hline 15 & rs809736 & 61329788 & $G$ & 0.32 & 0.38 & 1.32 & 0.224 & 0.22 & RORA & $8.19 E-06$ & 1.52 & $4.57 E-06$ & 1.49 & 0 \\
\hline
\end{tabular}

Abbreviations: BP, base position based on hg19; CHR, chromosome; OR, odds ratio; SNP, single nucleotide polymorphism; STAR*D, Sequenced Treatment Alternatives to Relieve Depression study. ${ }^{a}$ Garriock et al. (2010). ${ }^{3}$ 
of the current study and the STAR ${ }^{\star} \mathrm{D}$ study. These analyses were performed using PLINK v1.07. ${ }^{6}$ Written informed consent was obtained after providing a complete description of the study to the subjects. The ethics committees at the Fujita Health University, and the University of Occupational and Environmental Health approved this study.

Only one SNP (rs6127921: $105 \mathrm{~kb}$ away from bone morphogenetic protein 7 gene (BMP7)) showed a significant association with treatment response to SSRIs in our data set $(P=0.0098$, odds ratio $(\mathrm{OR})=0.59,95 \%$ confidence intervals: $0.39-0.88)$, with a similar effect size as the $\mathrm{STAR}^{\star} \mathrm{D}$ study $(P=3.45 \times$ $\left.10^{-6}, \quad \mathrm{OR}=0.61\right)$. The other two SNPs showed a lack of association (Table 1). In the subsequent meta-analysis of the BMP7 SNP, although genome-wide significance was not detected $\left(P=5 \times 10^{-8}\right)$, the significance level improved $\left(P=1.1 \times 10^{-7}, \quad I^{2}=0\right)$. These results indicate that $B M P 7$ is a potent candidate gene for influencing SSRI efficacy in MDD patients worldwide. Although the detailed mechanism of BMP7 on antidepressant action has been largely unknown, a recent study reported the reduced BMP7 expression level in locus coeruleus in MDD subjects. This post-mortem finding suggests that BMP7 has a possible role on brain noradrenergic systems in MDD, thus supporting the association between $B M P 7$ and MDD (or antidepressant action). ${ }^{7}$

A recent mega-analysis of three pharmacogenomic studies, including $\operatorname{STAR}^{\star} \mathrm{D}$, did not detect this BMP7 SNP in the top findings. ${ }^{5}$ Several reasons can be proposed for the discrepancy between the results of our study, the $\operatorname{STAR}^{\star} \mathrm{D}$ study and the mega-analysis. The main outcome of the mega-analysis ${ }^{5}$ was remission after treatment with several antidepressants (for example, SSRIs, nortriptyline and others), which was defined as a HAM-D score $<7$ points. In our data set (number of patients in remission $=84 \quad(37.5 \%)$, which was less than those examined in the mega-analysis $(41 \%)^{5}$ and the $\operatorname{STAR}^{\star} \mathrm{D}$ only $\left.(55 \%)^{3}\right)$, we failed to detect a significant association even with the BMP7 SNP $(P=0.19$, $\mathrm{OR}=0.77 \quad(95 \%$ confidence interval $0.51-$ 1.14)); thus, the uncontrolled bias in our study may have affected the results. In general, although there is a correlation between the categorical variables of 'responder' and 'remission,' several clinical or environmental factors are also likely to influence 'remission' more than 'responder', as 'remission' requires a more stringent level. Therefore, to interpret the analysis of 'remission', at least in our data set, greater attention to several confounding factors (for example, patients' characteristics in the social domain) will be needed.

In conclusion, our results suggested an association between a BMP7 SNP and SSRI treatment response, but further replication studies will be essential to obtain conclusive results.

\section{ACKNOWLEDGEMENTS}

This work was supported by research grants from a Health Labor Sciences Research Grant of the Ministry of Health Labor and Welfare of Japan; Grants-in-Aid for Scientific Research from the Ministry of Education, Culture, Sports, Science and Technology (MEXT) of Japan; Grants-in-Aid for Scientific Research on Innovative Areas (Comprehensive Brain Science Network) from MEXT of Japan and the Strategic Research Program for Brain Sciences of MEXT of Japan.
Kosei Esaki ${ }^{1}$, Kenji Kondo ${ }^{1}$, Masakazu Hatano ${ }^{1}$, Takeo Saito ${ }^{1}$, Taro Kishi ${ }^{1}$, Wakako Umene-Nakano ${ }^{2}$, Reiji Yoshimura ${ }^{2}$, Jun Nakamura ${ }^{2}$, Norio Ozaki ${ }^{3}$, Masashi Ikeda ${ }^{1}$ and Nakao Iwata ${ }^{1}$

${ }^{1}$ Department of Psychiatry, Fujita Health University School of Medicine, Aichi, Japan; ${ }^{2}$ Department of Psychiatry, University of Occupational and Environmental Health, Fukuoka, Japan and ${ }^{3}$ Department of Psychiatry, Nagoya University Graduate School of Medicine, Nagoya, Japan E-mail: ikeda-ma@fujita-hu.ac.jp

1 Cirulli, E. T. \& Goldstein, D. B. Uncovering the roles of rare variants in common disease through whole-genome sequencing. Nat. Rev. Genet. 11, 415-425 (2010).

2 Tansey, K. E., Guipponi, M., Hu, X., Domenici, E., Lewis, G., Malafosse, A. et al. Contribution of common genetic variants to antidepressant response. Biol Psychiatry 73, 679-682 (2013).

3 Garriock, H. A., Kraft, J. B., Shyn, S. I., Peters, E. J., Yokoyama, J. S., Jenkins, G. D. et al. A genomewide association study of citalopram response in major depressive disorder. Biol. Psychiatry 67, 133-138 (2010).

4 Kishi, T., Fukuo, Y., Yoshimura, R., Okochi, T., Kitajima, T., Naitoh, H. et al. Pharmacogenetic study of serotonin 6 receptor gene with antidepressant response in major depressive disorder in the Japanese population. Hum. Psychopharmacol. 25, 481-486 (2010).

5 GENDEP Investigators, MARS Investigators, STAR*D Investigators. Common genetic variation and antidepressant efficacy in major depressive disorder: a metaanalysis of three genome-wide pharmacogenetic studies. Am. J. Psychiatry 170, 207-217 (2013).

6 Purcell, S., Neale, B., Todd-Brown, K., Thomas, L., Ferreira, M. A., Bender, D. et al. PLINK: a tool set for whole-genome association and population-based linkage analyses. Am. J. Hum. Genet. 81, 559-575 (2007).

7 Ordway, G. A., Szebeni, A., Chandley, M. J., Stockmeier, C. A., Xiang, L., Newton, S. S. et al. Low gene expression of bone morphogenetic protein 7 in brainstem astrocytes in major depression. Int. J. Neuropsychopharmacol. 15, 855-868 (2012). 